\title{
Ophiopogonin B induces reactive oxygen species-dependent apoptosis through the Hippo pathway in nasopharyngeal carcinoma
}

\author{
WENHUI DONG ${ }^{1}$, QING DONG ${ }^{2}$ and HAIRUI DING ${ }^{3}$ \\ ${ }^{1}$ Department of Otorhinolaryngology, Weifang People's Hospital, Weifang, Shandong 261041; \\ ${ }^{2}$ Department of Operating Room, Weifang Yidu Central Hospital, Weifang, Shandong 262500; \\ ${ }^{3}$ Department of Emergency, Weifang People's Hospital, Weifang, Shandong 261041, P.R. China
}

Received December 21, 2020; Accepted May 13, 2021

DOI: $10.3892 / \mathrm{mmr} .2021 .12173$

\begin{abstract}
Nasopharyngeal carcinoma (NPC) is a common malignant tumor in South China and is characterized by a high death rate. Ophiopogonin B (OP-B) is a bioactive component of Radix Ophiopogon japonicus, which is frequently used in traditional Chinese medicine to treat cancer. The present study aimed to examine the anti-cancer properties of OP-B on NPC cells. Cell viability and cell proliferation were measured using MTT and EdU assays. Flow cytometry was used to measure cell apoptosis, reactive oxygen species and mitochondrial membrane potential. Western blotting was used to investigate the expression of apoptosis and Hippo signaling pathway proteins. OP-B inhibited the proliferation of NPC cells by inducing apoptosis and disturbing the mitochondrial integrity. OP-B enhanced ROS accumulation. In addition, OP-B promoted the expression of mammalian STE20-like kinase 1, large tumor suppressor 1 and phosphorylated yes-associated protein (YAP) and suppressed the expression of YAP and transcriptional enhanced associate domain in NPC cells. OP-B increased the expression of forkhead box transcription factor $\mathrm{O} 1$ in the nuclear fraction. In conclusion, OP-B has therapeutic potential and feasibility in the development of novel YAP inhibitors for NPC.
\end{abstract}

\section{Introduction}

Nasopharyngeal carcinoma (NPC) is associated with Epstein-Barr virus infection (1). In 2018, there were an estimated 129,079 new cases of NPC and the an estimated 72,987 deaths from NPC worldwide (2). Epidemiological trends have shown that the incidence of NPC has declined progressively and related mortality has reduced substantially

Correspondence to: Dr Hairui Ding, Department of Emergency, Weifang People's Hospital, 151 Guangwen Street, Kuiwen, Weifang, Shandong 261041, P.R. China

E-mail: hairuiding@126.com

Key words: nasopharyngeal carcinoma, ophiopogonin B, Hippo pathway in the past decade (3). Given the complex proximity of the nasopharynx, surgical excision is rarely used to treat primary NPC (4). For a long time, radiotherapy has been recognized as the mainstay for the treatment of NPC (5), but $\sim 50 \%$ of patients experienced recurrent tumor (6). Therefore, there is an urgent need to identify more effective agents.

Ophiopogonin B (OP-B) is one of the main active components of Radix Ophiopogonjaponicus (7). Studies have revealed that OP-B suppresses tumorigenesis and induces apoptosis in gastric (8), colorectal (9) and lung cancers (10). OP-B has been shown to regulate multiple cancer-related signaling mechanisms, including JNK/c-Jun (9), EPH Receptor A2/AKT (11), PI3K/AKT (12) and ERK signaling pathways (12). However, the anti-cancer function of OP-B and the underlying specific mechanisms remain to be elucidated.

The Hippo signaling pathway consists of a kinase cascade and transcription coactivators (13). Aberrant regulation of the Hippo pathway has been reported in several types of cancer, including NPC (14-17). However, no studies have reported on the anticancer effect of OP-B by regulating the Hippo pathway in NPC. The objective of the present study was to evaluate the effect of OP-B against NPC and investigate the underlying mechanisms.

\section{Materials and methods}

Cell culture. The present study chose human EBV positive NPC cell (C666-1) and EBV negative cells (HK1) (18). C666-1 and HK1 cells were purchased from the National Infrastructure of Cell Line Resource, Peking Union Medical College (Beijing, China). C666-1 and HK1 cells were cultured in Dulbecco's modified Eagle medium (HyClone; Cytiva) containing 10\% fetal bovine serum (HyClone; Cytiva). All cells were then incubated at $37^{\circ} \mathrm{C}$ in a humidified atmosphere of $5 \% \mathrm{CO}_{2}$

Ophiopogonin B (OP-B; purity of $\geq 97 \%$; Shanghai Tauto Biotech Co., Ltd.) was dissolved in dimethyl sulfoxide (DMSO; Sigma-Aldrich; Merck KGaA). For treatment of cells, OP-B was diluted in culture medium to a final concentration of 5,10 and $20 \mu \mathrm{M}(0.01 \% \mathrm{DMSO})$ at $25^{\circ} \mathrm{C}$ for $30 \mathrm{~min}$.

MTT assay. Cells were seeded $\left(5 \times 10^{4}\right.$ cells/well $)$ in 96-well plates and then OP-B $(5,10$ and $20 \mu \mathrm{M})$ was added for 
12, 24, 48 or 72 h. MTT (20 $\mu 1 ; 5$ mg/ml; Sigma-Aldrich; Merck KGaA) was added and incubated for another $4 \mathrm{~h}$ at $37^{\circ} \mathrm{C}$. Afterwards, $150 \mu \mathrm{l}$ dimethyl sulfoxide was added at room temperature for $10 \mathrm{~min}$. Absorbance was assessed at $490 \mathrm{~nm}$ using a microplate reader (BioTek Instruments, Inc.).

EdU assay. Cell proliferation was detected using the BeyoClick $^{\mathrm{TM}}$ EdU-594 detection kit (cat. no. C0078S; Beyotime Institute of Biotechnology). Briefly, following treatment with OP-B, cells were incubated with $50 \mathrm{mM}$ EdU for $2 \mathrm{~h}$ at $37^{\circ} \mathrm{C}$ and incubated with 4',6-diamidino-2-phenylindole for $30 \mathrm{~min}$ at $37^{\circ} \mathrm{C}$. After staining, images were photographed under a fluorescence microscope (Olympus).

Apoptosis analysis. The Annexin V-FITC/propidium iodide (PI) apoptosis detection kit was used to detect cell apoptosis. Briefly, following treatment with OP-B, cells were collected. $5 \mu \mathrm{l}$ Annexin V-FITC and $5 \mu \mathrm{l}$ PI were then added, mixed and incubated for $15 \mathrm{~min}$ at room temperature. Apoptosis was evaluated using a flow cytometer (BD Accuri C6 Plus; BD Biosciences) and FlowJo software (v10.6.2; FlowJo, LLC). The apoptotic rate was calculated as the percentage of early + late apoptotic cells.

Measurement of mitochondrial membrane potential (MMP). MMP was examined using the fluorescent probe 5,5',6,6-tetrachloro-1, 1',3,3'-tetraethyl-benzimidazolylcarbocyanine iodide (JC-1, Beyotime Institute of Biotechnology). Briefly, following treatment with OP-B, cells were incubated with JC-1 staining solution at $37^{\circ} \mathrm{C}$ for $20 \mathrm{~min}$. MMP was detected using flow cytometry as aforementioned (BD Biosciences).

Measurement of intracellular reactive oxygen species (ROS). Intracellular ROS were detected using an ROS assay kit (Nanjing Jiancheng Bioengineering Institute). In brief, following treatment with OP-B, $10 \mu \mathrm{M}$ 2,7-dichlorodihydrofluorescein diacetate (DCFH-DA) was added to the cells at $37^{\circ} \mathrm{C}$ for $20 \mathrm{~min}$. The median fluorescence intensity of ROS was measured using a flow cytometer as aforementioned (BD Biosciences).

Malondialdehyde (MDA) assay and superoxide dismutase (SOD) assay. MDA and SOD activity were assessed using MDA assay kit and SOD assay kit (Nanjing Jiancheng Bioengineering Institute).

Small interfering RNA (siRNA) transfection. The yes-associated protein (YAP) siRNA (sense: 5'-ACUUUUCGCUGCAAG UUGCUA-3'; antisense: 3'-GCAACUUGCAGCGAAAAG UUU-5'), control siRNA (sense: 5'-UUCUCCGAACGUGUC ACGUTT-3'; antisense: 3'-ACGUGACACGUUCGGAGA ATT-5') were synthesized by Shanghai GenePharma Co., Ltd. C666-1 and HK1 cells (10 6 cells/well) were seeded in six-well plates. The cells were transfected with $100 \mathrm{nM}$ of YAP siRNA or control siRNA using Lipofectamine ${ }^{\circledR} 2000$ (Invitrogen; Thermo Fisher Scientific, Inc.) at $37^{\circ} \mathrm{C}$, and the medium was changed for fresh after $6 \mathrm{~h}$. After $48 \mathrm{~h}$, the C666-1 and HK1 cells were harvested and exposed to OP-B $(5 \mu \mathrm{M})$ for $24 \mathrm{~h}$ at $37^{\circ} \mathrm{C}$.

Western blotting. C666-1 and HK1 cells $\left(1 \times 10^{6}\right)$ were harvested and lysed them with radioimmunoprecipitation assay buffer
(Beyotime Institute of Biotechnology). Total protein was quantified by using an Enhanced BCA Protein Assay kit (Beyotime Institute of Biotechnology). Total protein $(50 \mu \mathrm{g})$ was loaded on $12 \%$ sodium dodecyl sulphate polyacrylamide gel electrophoresis. Subsequently, the gel was transferred to a polyvinylidene fluoride membrane (Millipore). The membrane was blocked by using $5 \%$ non-fat milk for $1 \mathrm{~h}$ at $25^{\circ} \mathrm{C}$. After blocking, the membrane was incubated with anti-YAP $(1: 1,000$; cat. no. 4912), anti-phosphorylated (p-)YAP (S127; 1:1,000; cat. no. 4911), anti-mammalian sterile 20-like kinase 1 (MST1; 1:1,000; cat. no. 3682), anti-large tumor suppressor 1 (LATS1; 1:1,000; cat. no. 3477), anti-transcriptional enhanced associate domain 1 (TEAD1; 1:1,000; cat. no. 12292), anti-Bcl-2 (1:1,000; cat. no. 3498), anti-Bax (1:1,000; cat. no. 2774), anti-caspase-3 (1:1,000; cat. no. 9662), anti-cleaved caspase-3 (1:1,000; cat. no. 9661), anti-poly(ADP-ribose) polymerase (PARP; 1:1,000; cat. no. 9542), anti-cleaved-PARP (1:1,000; cat. no. 9545), anti-forkhead box transcription factor O1 (FOXO1; 1:1,000; cat. no. 2880), anti-p-FOXO1 (1:1,000; cat. no. 2486; all from Cell Signaling Technology, Inc.) and anti-glyceraldehyde-3-phosphate dehydrogenase (1:5,000; cat. no. P30008; Abmart Pharmaceutical Technology Co., Ltd.) overnight at $4^{\circ} \mathrm{C}$. Membranes were incubated with secondary antibody (1:5,000; cat. no. M21002, Abmart Pharmaceutical Technology Co., Ltd.) at room temperature for $2 \mathrm{~h}$. Protein bands were detected using a chemiluminescence kit and a gel imaging system (Tanon 2500; Tanon Science and Technology Co., Ltd.).

Statistical analysis. Statistical analyses were performed using GraphPad Prism 7 (GraphPad Software, Inc.). The data represented mean \pm standard deviation from three independent experiments. One-way ANOVA and Tukey's post hoc test were conducted to evaluate changes among groups. $\mathrm{P}<0.05$ was considered to indicate a statistically significant difference.

\section{Results}

$O P-B$ inhibits the proliferation of NPC cells. The chemical structure of OP-B is shown in Fig. 1A. The effect of OP-B on cell proliferation was investigated in C666-1 and HK1 cell lines. The MTT assay demonstrated that OP-B inhibited C666-1 and HK1 cells proliferation in a dose and time-dependent manner (Fig. 1B). As shown in Fig. 1C, OP-B effectively inhibited the proliferation of C666-1 and HK1 cells.

$O P-B$ induces apoptosis in NPC cells. Next, whether OP-B induced apoptosis was investigated. Flow cytometry assays were used to confirm that OP-B activated apoptosis in C666-1 and HK1 cells (Fig. 2A). Furthermore, OP-B increased the expression of Bax, cleaved-PARP and cleaved-caspase-3, whereas the expression of Bcl-2, PARP and caspase-3 was decreased by OP-B in C666-1 and HK1 cells (Fig. 2B). OP-B induced a concentration-dependent decrease in red/green fluorescence ratios in C666-1 and HK1 cells (Fig. 2C).

$O P-B$ induces ROS in NPC cells. ROS from mitochondria are related to cell apoptosis (19). Following treatment with OP-B, intracellular ROS levels were increased in C666-1 and HK1 cells (Fig. 3A). As shown in Fig. 3B and C, OP-B increased the MDA content and decreased SOD activity. 
A

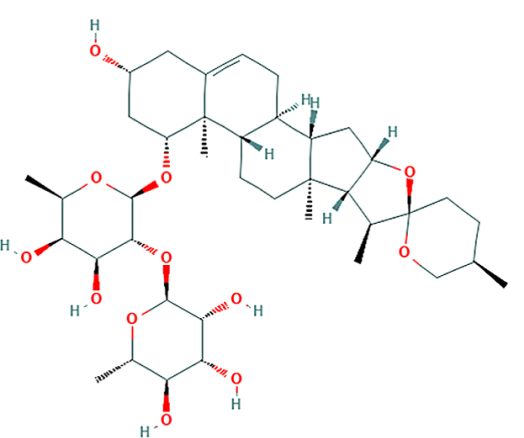

$\mathrm{B}$

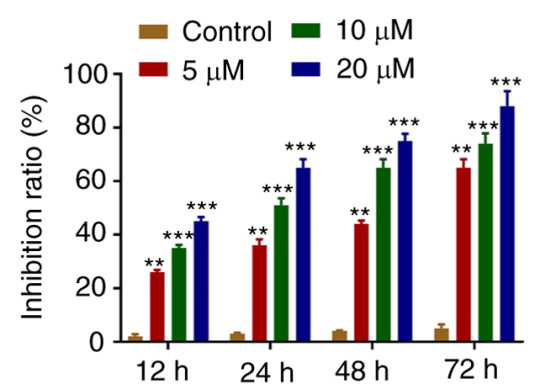

C666-1

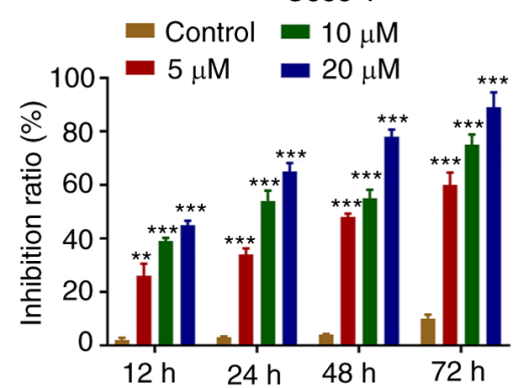

C
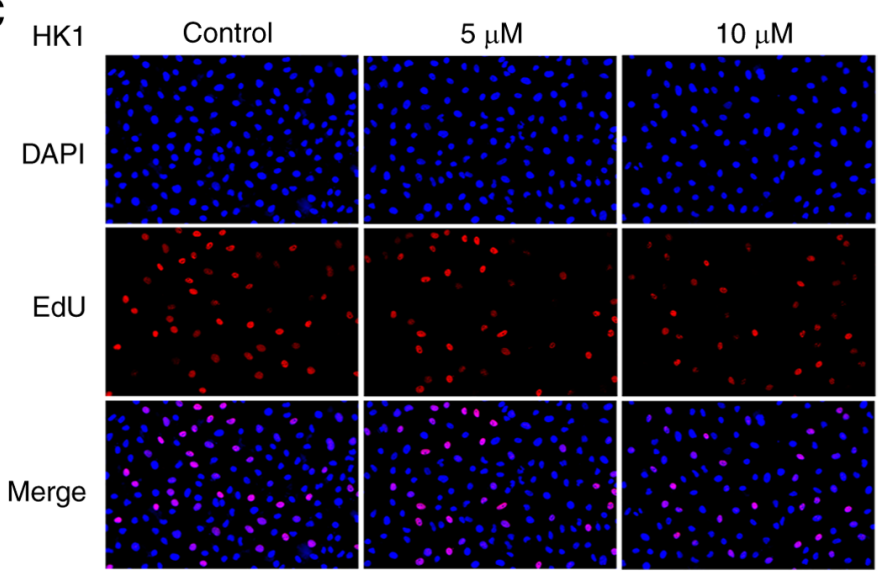

$20 \mu \mathrm{M}$
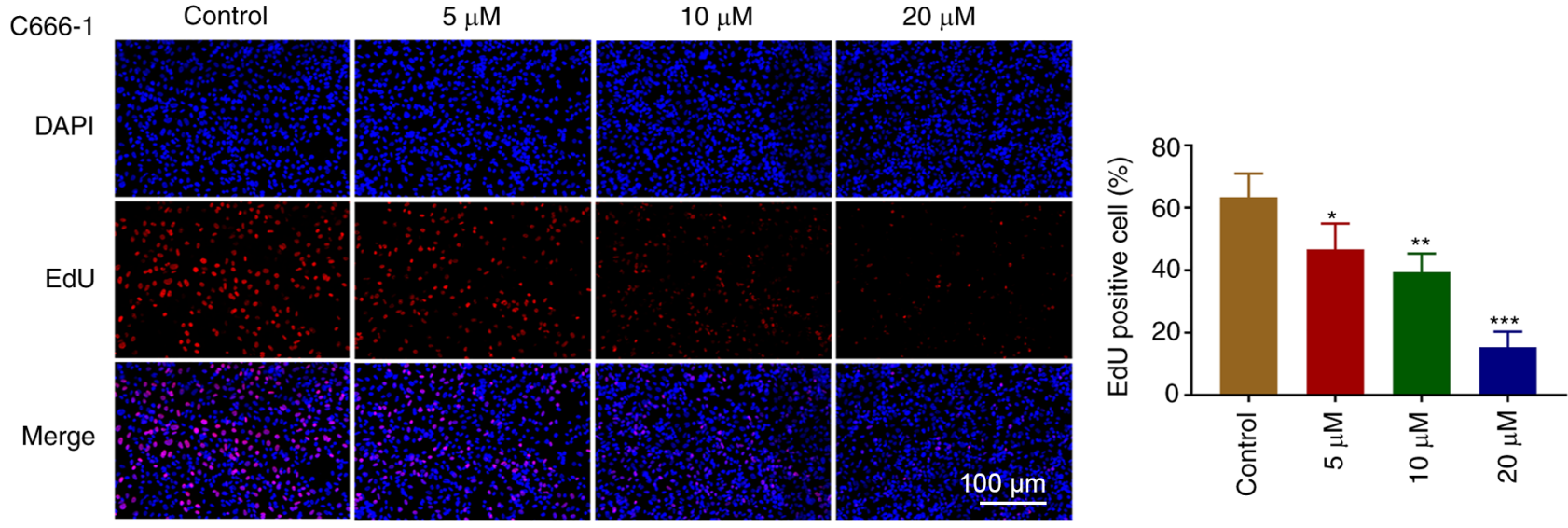

Figure 1. OP-B inhibits the proliferation of nasopharyngeal carcinoma cells. (A) Chemical structure of OP-B. (B) MTT was used to evaluate cell inhibition rate. (C) EdU assay was used to measure cell proliferation. ${ }^{*} \mathrm{P}<0.05 ;{ }^{* *} \mathrm{P}<0.01$; and ${ }^{* * * *} \mathrm{P}<0.001$ vs. control group. OP-B, Ophiopogonin $\mathrm{B}$.

$O P-B$ increases the expression of FOXO1 in the nuclear fraction. OP-B treatment decreased the ratio of $\mathrm{p}-\mathrm{FOXO} 1$ vs. total FOXO1 in C666-1 and HK1 cells (Fig. 4A). In addition, FOXO1 protein level decreased in cytosolic fraction and increased in the nuclear fraction following treatment with OP-B (Fig. 4B).

$O P-B$ regulates the Hippo-YAP signaling pathway in NPC cells. OP-B significantly increased the expression of MST1 and LATS1, increased the ratio of p-YAP vs. total YAP and decreased TEAD protein levels (Fig. 5). Therefore, the results suggested that OP-B inhibited NPC cells tumorigenesis through the regulation of the Hippo signaling pathway.

Dysfunction of hippo-YAP signaling increases the antitumor function of $O P-B$. siYAP was transfected into C666-1 and
HK1 cells and the cell proliferation and apoptosis ability of these two NPC cell lines detected. The expression of YAP decreased rapidly due to OP-B treatment, YAP knockdown significantly reduced YAP expression (Fig. 6A). YAP knockdown promoted the inhibitory effect of OP-B on the proliferation of C666-1 and HK1 cells (Fig. 6B). In addition, YAP knockdown induced the enhancing effect of OP-B on apoptosis in C666-1 and HK1 cells (Fig. 6C).

\section{Discussion}

The current study revealed that OP-B exerted its anti-cancer effects by inhibiting cell proliferation, inducing cell apoptosis and regulating the Hippo signaling pathway.

The mitochondrial pathway is a crucial mechanism of ophiopogonin-mediated cell death in gastric cancer 


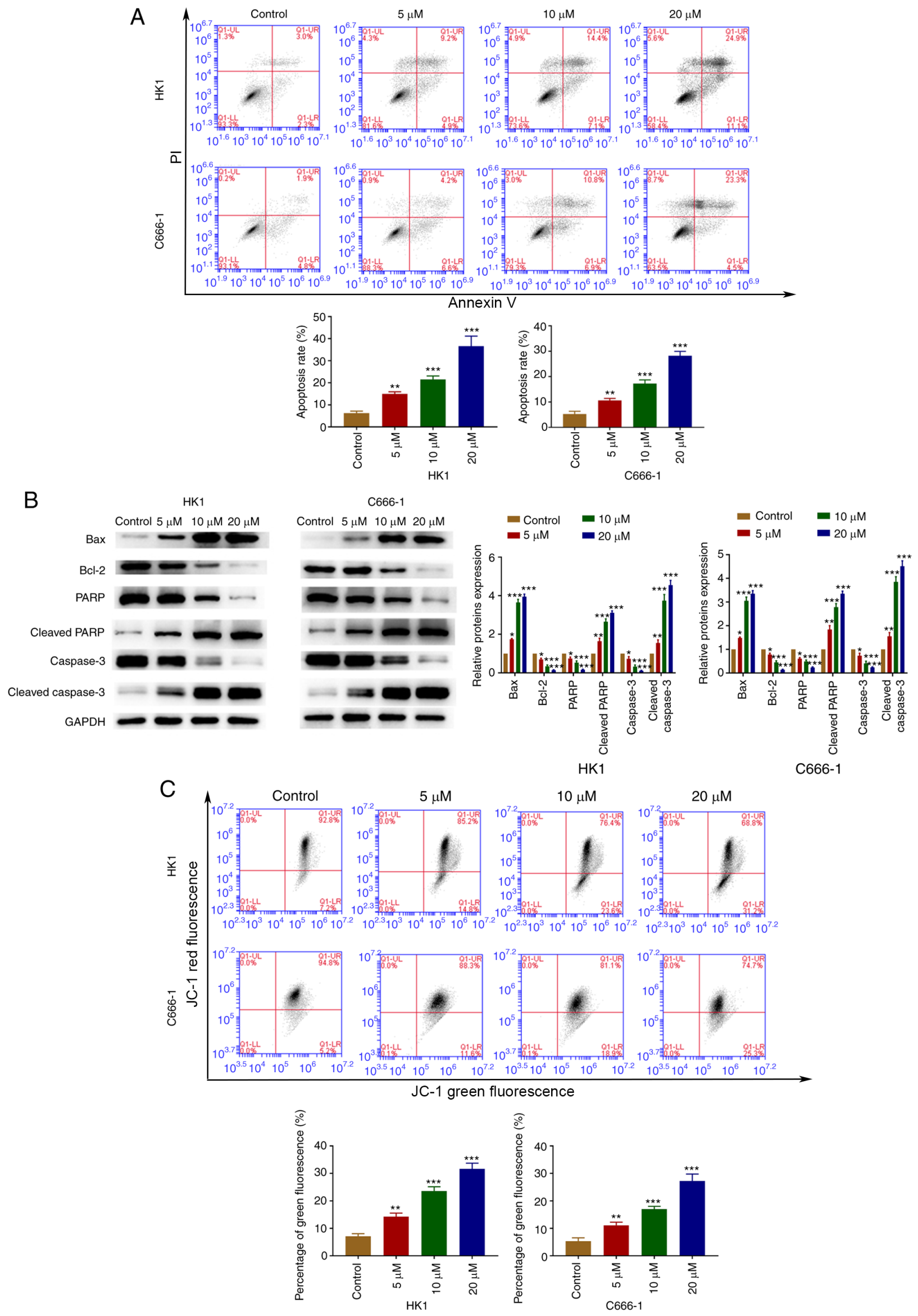

Figure 2. OP-B induced apoptosis of nasopharyngeal carcinoma cells. (A) Annexin V/PI analysis was used to detected OP-B induced apoptosis rates. (B) The expression of Bax, Bcl-2, cleaved caspase-3, caspase-3, cleaved PARP1 and PARP1 was detected by western blotting. (C) Mitochondrial membrane potential was evaluated using JC-1 staining. " $\mathrm{P}<0.05 ;{ }^{* *} \mathrm{P}<0.01$; and ${ }^{* * * *} \mathrm{P}<0.001$ vs. control group. OP-B, Ophiopogonin B; JC-1, 5,5',6,6-tetrachloro-1, 1',3,3'-tetraethylbenzimidazolylcarbocyanine iodide. 

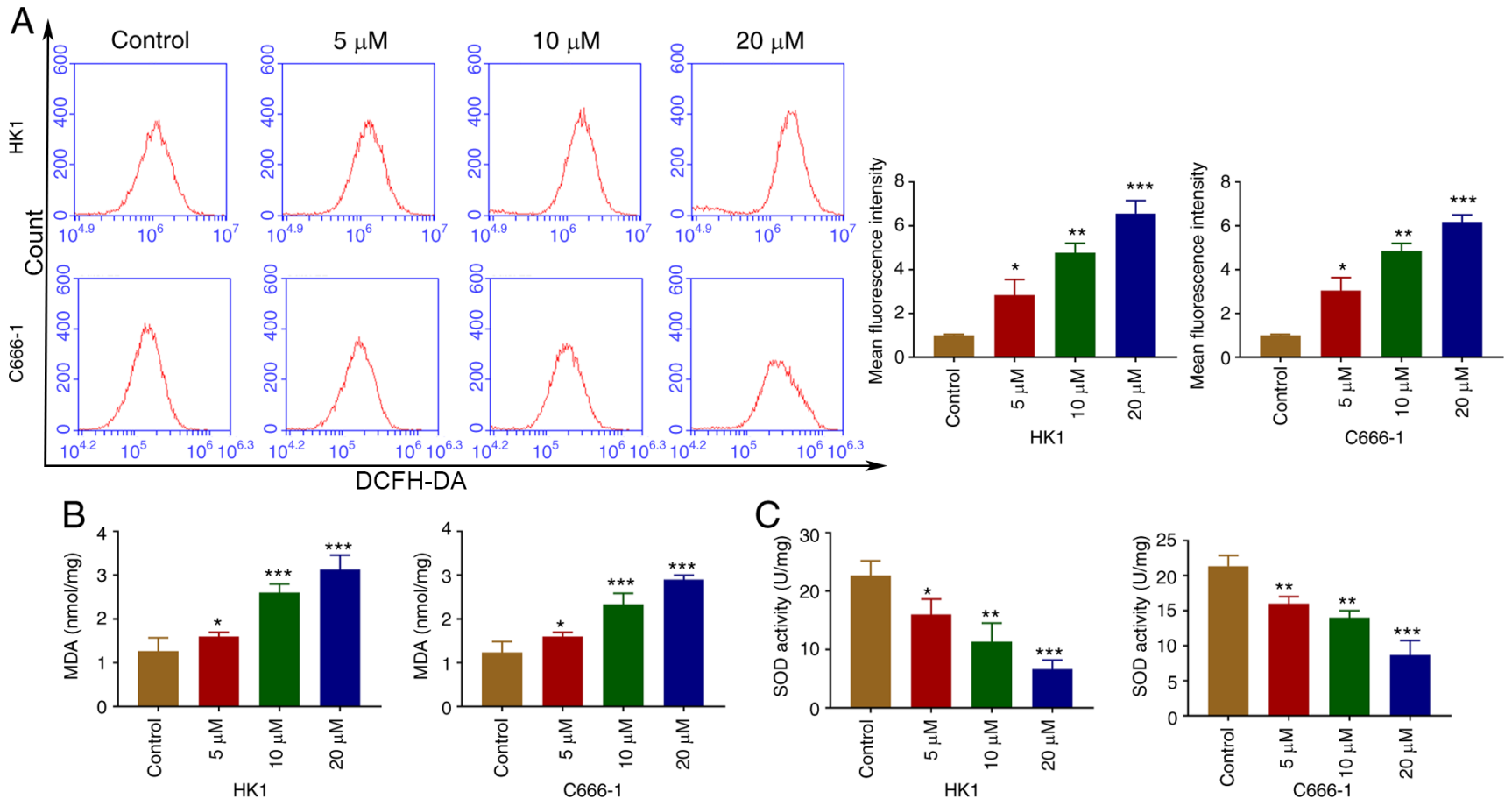

Figure 3. OP-B induced ROS generation in NPC. (A) ROS generation was detected by flow cytometry. Effect of OP-B on (B) MDA content and (C) SOD activities in NPC cells. ${ }^{*} \mathrm{P}<0.05 ;{ }^{* *} \mathrm{P}<0.01$; and ${ }^{* * *} \mathrm{P}<0.001$ vs. control group. OP-B, Ophiopogonin B; ROS, reactive oxygen species; NPC, nasopharyngeal carcinoma cells; MDA, malondialdehyde; SOD, superoxide dismutase.
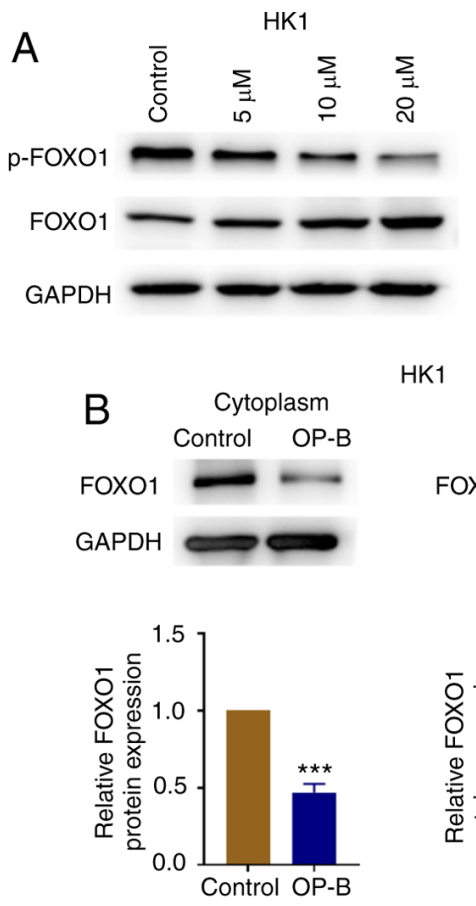

HK1

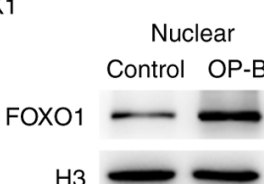

$\mathrm{H} 3$
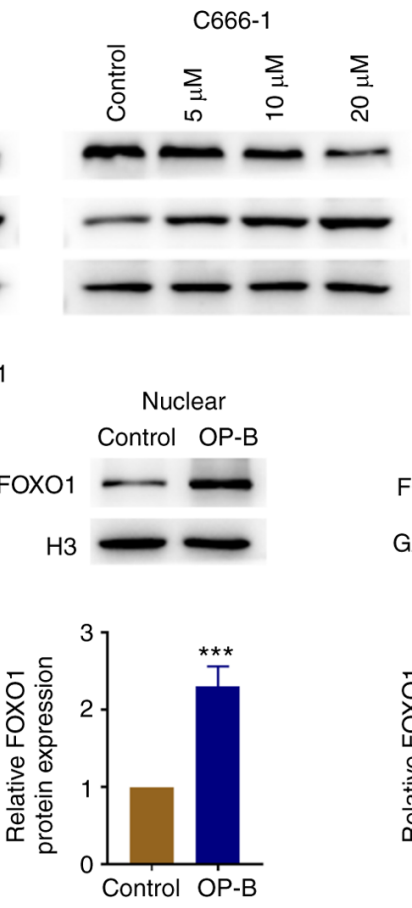

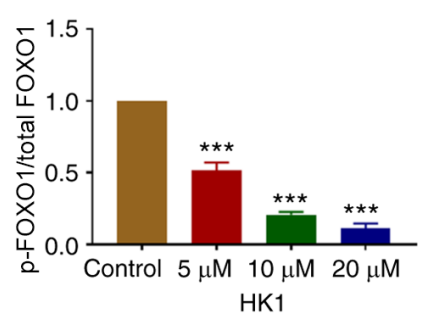

C666-1

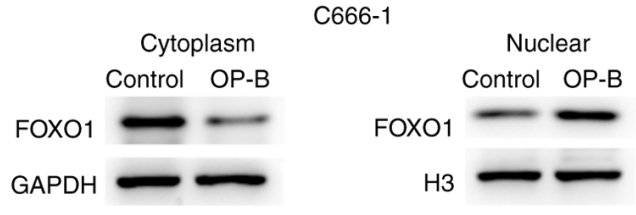

Figure 4. OP-B increases the expression of FOXO1 in nucleus. (A) The expression of p-FOXO1 and FOXO1 protein was evaluated by western blotting. (B) The expression of FOXO1 protein in nucleus and cytoplasm was separately analyzed by western blotting. ${ }^{*} \mathrm{P}<0.05 ;{ }^{* * *} \mathrm{P}<0.01$; and ${ }^{* * * *} \mathrm{P}<0.001$ vs. control group. OP-B, Ophiopogonin B; p-, phosphorylated; FOXO1, forkhead box transcription factor O1.

and prostate cancer $(8,20)$. The balance of proapoptotic protein (Bax) and antiapoptotic protein (Bcl-2) maintains a healthy survival/death balance in cells (21). Caspase-3 is a critical molecule for stimulating cancer apoptosis (22), which is activated by pro-apoptotic factors (23). The present study found that OP-B increased the $\mathrm{Bax} / \mathrm{Bcl}-2$ ratio and activated caspase-3 level. PARP could be cleaved by caspase-3 during apoptosis (24) and was also clearly detected following OP-B treatment in NPC cells. Meanwhile, suppression of MMP was found in the process of OP-B-induced NPC cell apoptosis, 
$\mathrm{HK}$
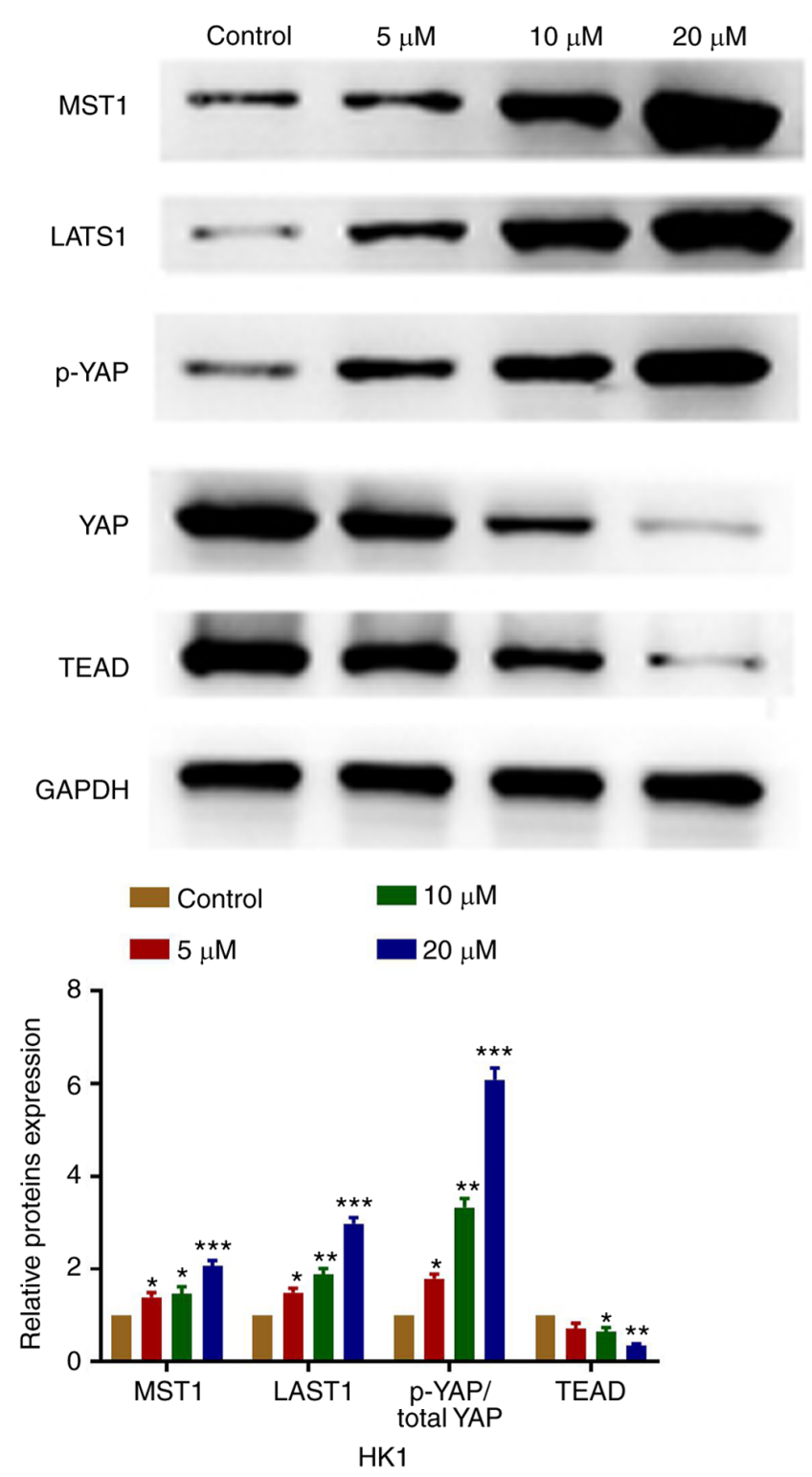

C666-1
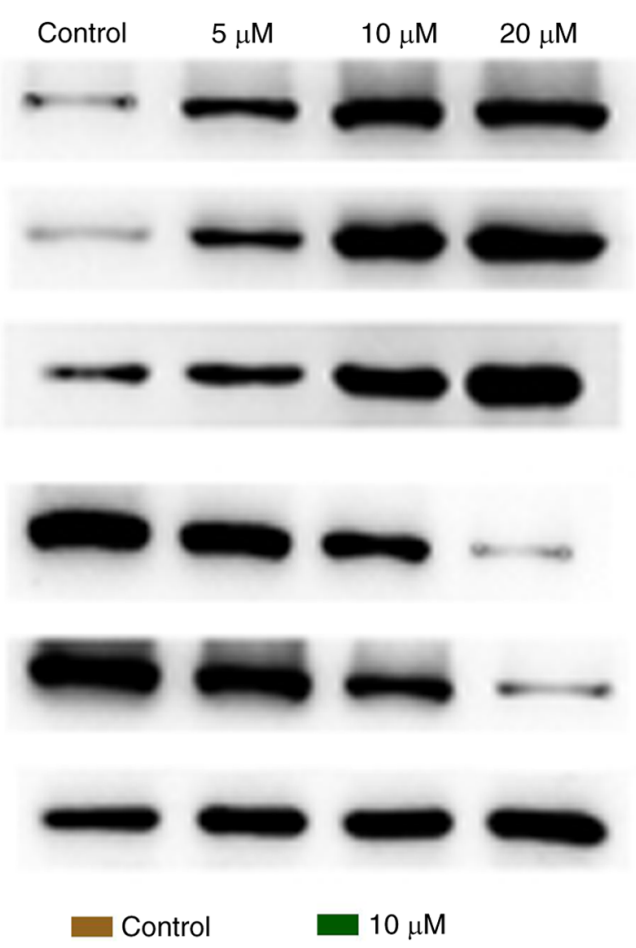

$5 \mu \mathrm{M} \quad 20 \mu \mathrm{M}$

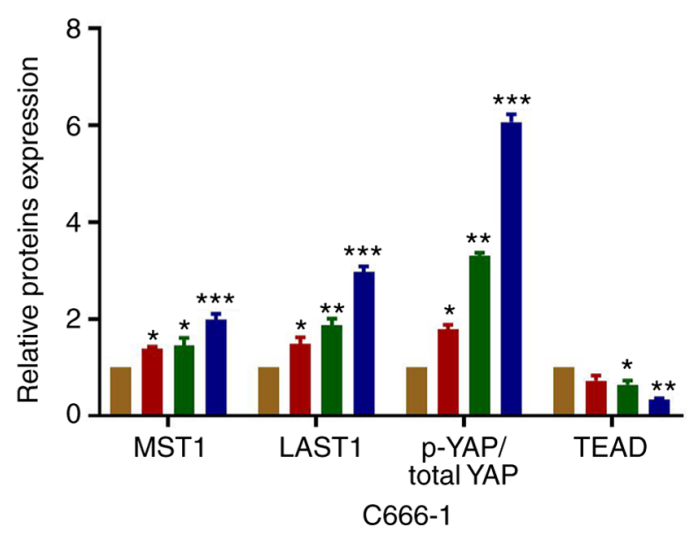

Figure 5. OP-B regulates the Hippo-YAP pathway in nasopharyngeal carcinoma cells. The expression of the Hippo-YAP pathway proteins was evaluated by western blotting. ${ }^{*} \mathrm{P}<0.05 ;{ }^{* *} \mathrm{P}<0.01$; and ${ }^{* * *} \mathrm{P}<0.001$ vs. control group. OP-B, Ophiopogonin B; p-, phosphorylated; YAP; yes-associated protein; MST1, mammalian sterile 20-like kinase 1; LATS1, large tumor suppressor 1; p-, phosphorylated; TEAD, transcriptional enhanced associate domain 1.

which was consistent with results of a previous study (8). This finding indicated that the mitochondria-mediated apoptotic pathway contributes to the process of OP-B-induced apoptosis in NPC cells.

The mitochondria of cancer cells overproduce ROS (25). ROS induce DNA-damage and cell death (26). The generation of ROS and reduction in MMP has been reported to be important in triggering apoptosis (27). Natural products have attracted attention as good candidate chemotherapeutic drugs for cancer therapies, due to their ability to maintain oxidative metabolism with minimal toxicity (28). For example, plumbagin is known to induce apoptosis in lung cancer through ROS production (29). OP-B participates in ROS generation in the gastric cancer cells (8). SOD is an antioxidant that removes superoxide radicals (30). It has been reported that targeting SOD is a promising approach to selectively kill cancer cells (31). The present study found that OP-B accelerated mitochondrial ROS production, decreased SOD levels and promoted apoptotic cell death in NPC cells.

FOXO1 serves an important role in cell proliferation and chemosensitivity in NPC cells (32). The results of the present study are in accordance with previous findings that trifluoperazine increases the expression of FOXO1 in the nucleus and enhances the expression of Bax, but decreases the expression of Bcl-2 (33).

The Hippo pathway was originally found in Drosophila melanogaster (34). It is generally accepted that the highly-conserved Hippo pathway serves a vital role in maintaining tissue and organ size, stem cell and tumorigenesis (35-37). YAP and PDZ-binding motif (TAZ) actively promote cell proliferation through a transcriptional program mediated by TEAD family transcription factors (38). Mechanistically, YAP/TAZ depletion diminishes 

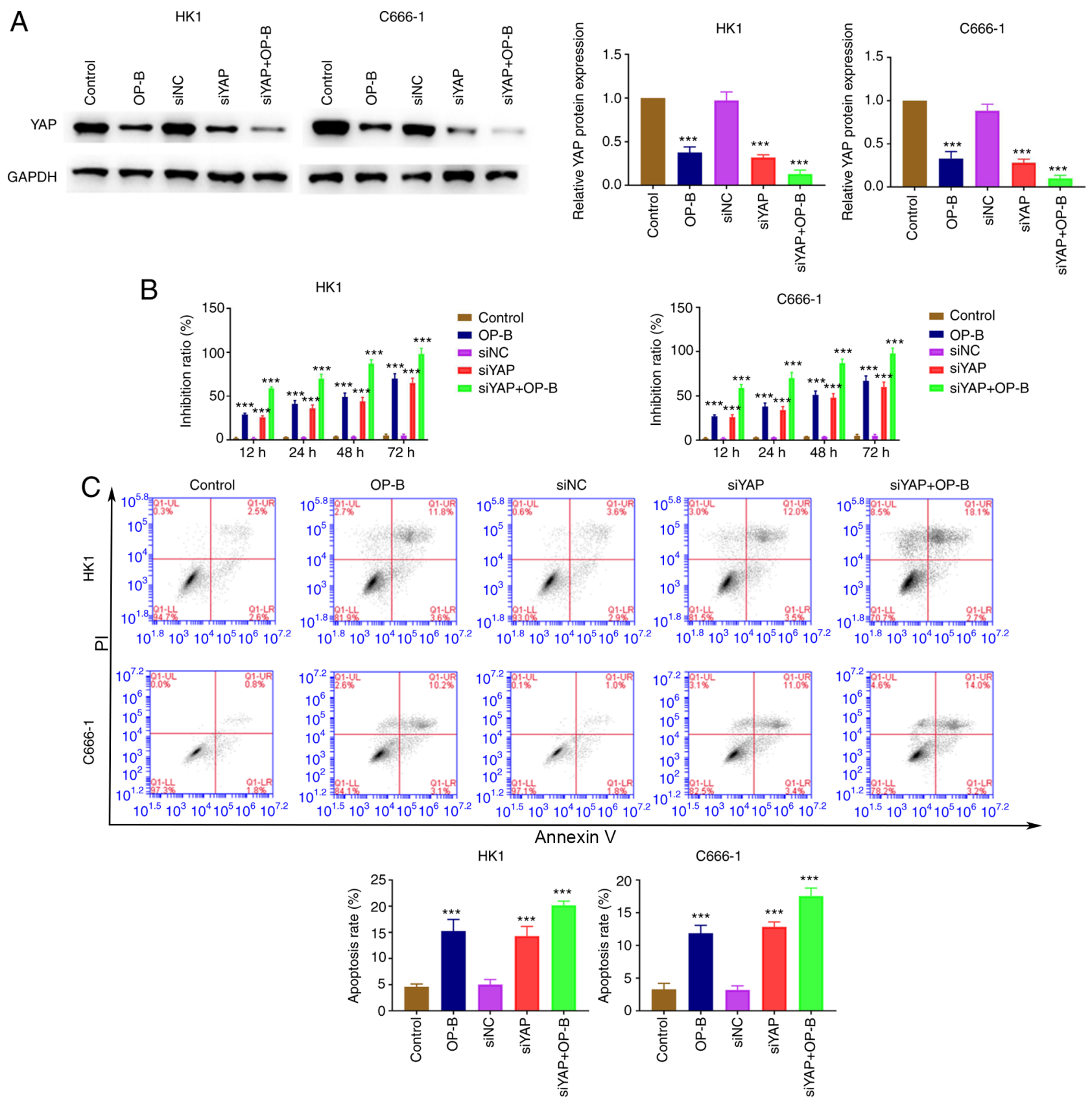

Figure 6. Knockdown of YAP increased the antitumor function of OP-B in nasopharyngeal carcinoma cells. (A) The expression of YAP protein was evaluated by western blotting. (B) MTT was used to evaluate cell inhibition rate. (C) Annexin V/PI analysis was used to measure the proapoptotic effect. ${ }^{* * *} \mathrm{P}<0001$ vs. control group. YAP; yes-associated protein; OP-B, Ophiopogonin B; si, short interfering; NC, negative control.

glycolysis-dependent proliferation and increases mitochondrial respiration and ROS production, resulting in oxidative stress-induced cell death (39). In NPC, the Hippo pathway has been found to be dysregulated. Li et al (40) reported that the Hippo pathway attenuates the sensitivity of NPC cells to cisplatin by inducing epithelial-mesenchymal transition. Elevated expression of YAP, a Hippo pathway effector, is observed in NPC (41). Overexpression of YAP rescues the effect of testis-associated oncogenic lncRNA knockdown on NPC cell stemness and sensitivity of NPC cells to cisplatin (42). In the present study, OP-B markedly increased the activation of Hippo pathway components, such as MST1, LATS1 and phosphorylated YAP. Additionally, the protein expression of YAP and TEAD was decreased in NPC cells following treatment with OP-B. Previous studies have shown the LATS-mediated phosphorylation of YAP at Ser127 and its nuclear localization (43-45). Moreover, YAP knockdown promoted the inhibitory effect of OP-B on proliferation and induced the effect of OP-B on apoptosis in C666-1 and HK1 cells. This result is partly consistent with a recent study that indicates that artemisinin inhibits hepatocellular carcinoma cell proliferation, migration and invasion by suppressing Hippo signaling (46). The results of the present study suggested that OP-B regulated Hippo signaling pathway in NPC cells.

The present study indicated that OP-B inhibited NPC cells survival by activating mitochondria-mediated apoptosis via the Hippo pathway. OP-B appears to be a potential therapeutic agent for NPC patients. 


\section{Acknowledgements}

Not applicable.

\section{Funding}

No funding was received.

\section{Availability of data and materials}

The datasets used and/or analyzed during the current study are available from the corresponding author on reasonable request.

\section{Authors' contributions}

WD and HD designed the study and performed the research; QD and HD analyzed the data and wrote the manuscript. WD and HD confirm the authenticity of all the raw data. All authors read and approved the final manuscript and agree to be accountable for all aspects of the work in ensuring that the accuracy or integrity of any part of the work are appropriately investigated and resolved.

\section{Ethics approval and consent to participate}

Not applicable.

\section{Patient consent for publication}

Not applicable.

\section{Competing interests}

The authors declare that they have no competing interests.

\section{References}

1. Glastonbury CM: Head and neck squamous cell cancer: Approach to staging and surveillance. In: Diseases of the Brain, Head and Neck, Spine 2020-2023: Diagnostic Imaging [Internet]. Hodler J, Kubik-Huch RJ and von Schulthess GK (eds.). Cham $(\mathrm{CH})$, Springer, Chapter 17, 2020.

2. Bray F, Ferlay J, Soerjomataram I, Siegel RL, Torre LA and Jemal A: Global cancer statistics 2018: GLOBOCAN estimates of incidence and mortality worldwide for 36 cancers in 185 countries. CA Cancer J Clin 68: 394-424, 2018.

3. Chen YP, Chan ATC, Le QT, Blanchard P, Sun Y and Ma J: Nasopharyngeal carcinoma. Lancet 394: 64-80, 2019.

4. Lee HM, Okuda KS, González FE and Patel V: Current perspectives on nasopharyngeal carcinoma. Adv Exp Med Biol 1164: 11-34, 2019.

5. Sun XS, Li XY, Chen QY, Tang LQ and Mai HQ: Future of radiotherapy in nasopharyngeal carcinoma. Br J Radiol 92: 9, 2019.

6. Caponigro F, Longo F, Ionna F and Perri F: Treatment approaches to nasopharyngeal carcinoma: A review. Anticancer Drugs 21: 471-477, 2010

7. Chen MH, Chen XJ, Wang M, Lin LG and Wang YT: Ophiopogon japonicus-A phytochemical, ethnomedicinal and pharmacological review. J Ethnopharmacol 181: 193-213, 2016.

8. Zhang W, Zhang Q, Jiang Y, Li F and Xin H: Effects of ophiopogonin B on the proliferation and apoptosis of SGC-7901 human gastric cancer cells. Mol Med Rep 13: 4981-4986, 2016.

9. Gao GY, Ma J, Lu P, Jiang X and Chang C: Ophiopogonin B induces the autophagy and apoptosis of colon cancer cells by activating JNK/c-Jun signaling pathway. Biomed Pharmacother 108: 1208-1215, 2018.
10. Hu C, Jiang R, Cheng Z, Lu Y, Gu L, Li H,Li L, Gao Q, Chen M and Zhang X: Ophiopogonin-B Suppresses Epithelial-mesenchymal transition in human lung adenocarcinoma cells via the Linc00668/miR-432-5p/EMT axis. J Cancer 10: 2849-2856, 2019.

11. Chen M, Hu C, Guo Y, Jiang R, Jiang H, Zhou Y, Fu H, Wu M and Zhang X: Ophiopogonin B suppresses the metastasis and angiogenesis of A549 cells in vitro and in vivo by inhibiting the EphA2/Akt signaling pathway. Oncol Rep 40: 1339-1347, 2018.

12. Chen M, Du Y, Qui M, Wang M, Chen K, Huang Z, Jiang M, Xiong F, Chen J, Zhou J, et al: Ophiopogonin B-induced autophagy in non-small cell lung cancer cells via inhibition of the PI3K/Akt signaling pathway. Oncol Rep 29: 430-436, 2013.

13. Pan D: The hippo signaling pathway in development and cancer. Dev Cell 19: 491-505, 2010.

14. Zhang Y, Wang M, Xu X, Liu Y and Xiao C: Matrine promotes apoptosis in SW480 colorectal cancer cells via elevating MIEF1-related mitochondrial division in a manner dependent on LATS2-Hippo pathway. J Cell Physiol 234: 22731-22741, 2019.

15. Kim SH, Jin H, Meng RY, Kim DY, Liu YC, Chai OH, Park BH and Kim SM: Activating hippo pathway via Rassf1 by ursolic acid suppresses the tumorigenesis of gastric cancer. Int J Mol Sci 20: 4709, 2019

16. Wang L, Wang J, Cao Y, Li W, Wang Y, Xu J and Xu G: Molecular evidence for better efficacy of hypocrellin A and oleanolic acid combination in suppression of HCC growth. Eur J Pharmacol 842: 281-290, 2019.

17. Zhu Y, He D, Bo H, Liu Z, Xiao M, Xiang L, Zhou J, Liu Y, Liu X, Gong L, et al: The MRVI1-AS1/ATF3 signaling loop sensitizes nasopharyngeal cancer cells to paclitaxel by regulating the Hippo-TAZ pathway. Oncogene 38: 6065-6081, 2019.

18. Wu RW, Chu ES, Huang Z, Xu CS, Ip CW and Yow CM: FosPeg PDT alters the EBV miRNAs and LMP1 protein expression in EBV positive nasopharyngeal carcinoma cells. J Photochem Photobiol B 127: 114-122, 2013.

19. Chong SJ, Low IC and Pervaiz S: Mitochondrial ROS and involvement of $\mathrm{Bcl}-2$ as a mitochondrial ROS regulator. Mitochondrion 19: 39-48, 2014.

20. Lu Z, Wang H, Zhu M, Song W, Wang J, Wu C, Kong Y, Guo J, Li N, Liu J, et al: Ophiopogonin D', a natural product from radix ophiopogonis, induces in vitro and in vivo RIPK1-dependent and caspase-independent apoptotic death in androgen-independent human prostate cancer cells. Front Pharmacol 9: 432, 2018.

21. Hassan M, Watari H, AbuAlmaaty A, Ohba Y and Sakuragi N: Apoptosis and molecular targeting therapy in cancer. Biomed Res Int 2014: 150845, 2014.

22. Crowley LC and Waterhouse NJ: Detecting cleaved caspase-3 in apoptotic cells by flow cytometry. Cold Spring Harb Protoc: Nov 1, 2016 (Epub ahead of print). doi: 10.1101/pdb.prot087312.

23. Li J, Wu Y, Wang D, Zou L, Fu C, Zhang J and Leung GP: Oridonin synergistically enhances the anti-tumor efficacy of doxorubicin against aggressive breast cancer via pro-apoptotic and anti-angiogenic effects. Pharmacol Res 146: 104313, 2019.

24. Boulares AH, Yakovlev AG, Ivanova V, Stoica BA, Wang G, Iyer S and Smulson M: Role of poly(ADP-ribose) polymerase (PARP) cleavage in apoptosis. Caspase 3-resistant PARP mutant increases rates of apoptosis in transfected cells. J Biol Chem 274: 22932-22940, 1999.

25. Yang Y, Karakhanova S, Hartwig W, D'Haese JG, Philippov PP, Werner J and Bazhin AV: Mitochondria and mitochondrial ROS in cancer: Novel targets for anticancer therapy. J Cell Physiol 231: 2570-2581, 2016.

26. Kocyigit A and Guler EM: Curcumin induce DNA damage and apoptosis through generation of reactive oxygen species and reducing mitochondrial membrane potential in melanoma cancer cells. Cell Mol Biol 63: 97-105, 2017.

27. He J, Wei W, Yang Q and Wang Y: Phillygenin exerts in vitro and in vivo antitumor effects in drug-resistant human esophageal cancer cells by inducing mitochondrial-mediated apoptosis, ROS generation and inhibition of the nuclear factor kappa B NF- $\kappa$ B signalling pathway. Med Sci Monit 25: 739-745, 2019.

28. NavaneethaKrishnan S, Rosales JL and Lee KY: ROS-mediated cancer cell killing through dietary phytochemicals. Oxid Med Cell Longev 2019: 9051542, 2019.

29. Tripathi SK, Rengasamy KRR and Biswal BK: Plumbagin engenders apoptosis in lung cancer cells via caspase-9 activation and targeting mitochondrial-mediated ROS induction. Arch Pharm Res 43: 242-256, 2020. 
30. He L, He T, Farrar S, Ji L, Liu T and Ma X: Antioxidants maintain cellular redox homeostasis by elimination of reactive oxygen species. Cell Physiol Biochem 44: 532-553, 2017.

31. Huang P, Feng L, Oldham EA, Keating MJ and Plunkett W: Superoxide dismutase as a target for the selective killing of cancer cells. Nature 407: 390-395, 2000.

32. Zhao M, Luo R, Liu Y, Gao L, Fu Z, Fu Q, Luo X, Chen Y, Deng X, Liang Z, et al: miR-3188 regulates nasopharyngeal carcinoma proliferation and chemosensitivity through a FOXO1-modulated positive feedback loop with mTOR-p-PI3K/AKT-c-JUN. Nat Commun 7: 11309, 2016

33. Jiang J, Huang Z, Chen X, Luo R, Cai H, Wang H, Zhang H, Sun $\mathrm{T}$ and Zhang Y: Trifluoperazine activates FOXO1-related signals to inhibit tumor growth in hepatocellular carcinoma. DNA Cell Biol 36: 813-821, 2017.

34. Meng Z, Moroishi T and Guan KL: Mechanisms of Hippo pathway regulation. Genes Dev 30: 1-17, 2016.

35. Dong L, Lin F, Wu W, Liu Y and Huang W: Verteporfin inhibits YAP-induced bladder cancer cell growth and invasion via Hippo signaling pathway. Int J Med Sci 15: 645-652, 2018.

36. Hsu PC, Jablons DM, Yang CT and You L: Epidermal Growth Factor Receptor (EGFR) Pathway, Yes-Associated Protein (YAP) and the Regulation of Programmed Death-Ligand 1 (PD-L1) in Non-Small Cell Lung Cancer (NSCLC). Int J Mol Sci 20: 3821, 2019.

37. Choi W, Kim J, Park J, Lee DH, Hwang D, Kim JH, Ashktorab H, Smoot D, Kim SY, Choi C, et al: YAP/TAZ initiates gastric tumorigenesis via upregulation of MYC. Cancer Res 78 3306-3320, 2018.

38. Koo JH and Guan KL: Interplay between YAP/TAZ and Metabolism. Cell Metab 28: 196-206, 2018.

39. White SM, Avantaggiati ML, Nemazanyy I, Di Poto C, Yang Y, Pende M, Gibney GT, Ressom HW, Field J, Atkins MB and Yi C: YAP/TAZ inhibition induces metabolic and signaling rewiring resulting in targetable vulnerabilities in NF2-deficient tumor cells. Dev Cell 49: 425-443.e9, 2019.
40. Li S, Zhang X, Zhang R, Liang Z, Liao W, Du Z, Gao C, Liu F, Fan $\mathrm{Y}$ and Hong H: Hippo pathway contributes to cisplatin resistant-induced EMT in nasopharyngeal carcinoma cells. Cell Cycle 16: 1601-1610, 2017

41. Song L, Tang H, Liao W, Luo X, Li Y, Chen T and Zhang X FOXC2 positively regulates YAP signaling and promotes the glycolysis of nasopharyngeal carcinoma. Exp Cell Res 357: 17-24, 2017.

42. Gao L, Cheng XL and Cao H: LncRNA THOR attenuates cisplatin sensitivity of nasopharyngeal carcinoma cells via enhancing cells stemness. Biochimie 152: 63-72, 2018.

43. Suemura S, Kodama T, Myojin Y, Yamada R, Shigekawa M, Hikita H, Sakamori R, Tatsumi T and Takehara T: CRISPR Loss-of-Function screen identifies the hippo signaling pathway as the mediator of regorafenib efficacy in hepatocellular carcinoma. Cancers 11: 1362, 2019.

44. Ye XY, Luo QQ, Xu YH, Tang NW, Niu XM, Li ZM, Shen SP, Lu S and Chen ZW: 17-AAG suppresses growth and invasion of lung adenocarcinoma cells via regulation of the LATS1/YAP pathway. J Cell Mol Med 19: 651-663, 2015.

45. Han Y, Tang Z, Zhao Y, Li Q and Wang E: TNFAIP8 regulates Hippo pathway through interacting with LATS1 to promote cell proliferation and invasion in lung cancer. Mol Carcinog 57: 159-166, 2018.

46. Li Y, Lu J, Chen Q, Han S, Shao H, Chen P, Jin Q, Yang M, Shangguan F, Fei M, et al: Artemisinin suppresses hepatocellular carcinoma cell growth, migration and invasion by targeting cellular bioenergetics and Hippo-YAP signaling. Arch Toxicol 93: 3367-3383, 2019.

This work is licensed under a Creative Commons Attribution-NonCommercial-NoDerivatives 4.0 International (CC BY-NC-ND 4.0) License. 